\section{Images in \\ Cardiovascular Medicine \\ Check for updates}

\title{
Coronary Angioscopy Findings before and after Excimer Laser Coronary Angioplasty for Bare-Metal Stent In-Stent Restenosis
}

\author{
Shinichiro Masuda (D, MD', Takashi Shibui (D, MD', Sho Nagamine ${ }^{1}$, MD', $^{1}$ \\ Takaaki Tsuchiyama (D, MD', and Takashi Ashikaga, MD, PhD² \\ 'Department of Cardiology, Tokyo Metropolitan Hiroo Hospital, Tokyo, Japan \\ ${ }^{2}$ Department of Cardiology, Japanese Red Cross Musashino Hospital, Tokyo, Japan
}

Received: Jan 7, 2019

Revised: Mar 5, 2019

Accepted: Mar 25, 2019

\section{Correspondence to Shinichiro Masuda, MD \\ Department of Cardiology, Tokyo Metropolitan Hiroo Hospital, 2-34-10, Ebisu, Shibuya-ku, Tokyo 150-0013, Japan. \\ E-mail: giga627@yahoo.co.jp}

Copyright $($ C 2019. The Korean Society of Cardiology

This is an Open Access article distributed under the terms of the Creative Commons Attribution Non-Commercial License (https:// creativecommons.org/licenses/by-nc/4.0) which permits unrestricted noncommercial use, distribution, and reproduction in any medium, provided the original work is properly cited.

ORCID iDs

Shinichiro Masuda (D)

https://orcid.org/0000-0002-2791-8538 Takashi Shibui (D)

https://orcid.org/0000-0002-0676-5595 Sho Nagamine (D)

https://orcid.org/0000-0002-3232-8014 Takaaki Tsuchiyama (D)

https://orcid.org/0000-0002-6923-9832

Conflict of Interest

The authors have no financial conflicts of interest.

\section{Author Contributions}

Conceptualization: Masuda S, Shibui T; Data curation: Masuda S, Nagamine S, Tsuchiyama T; Methodology: Masuda S, Shibui T; Writing - original draft: Masuda S; Writing - review \& editing: Tsuchiyama T, Ashikaga T.
A 59-year-old man who underwent bare-metal stent implantation in the left-anterior descending artery (LAD) 15 years previously was admitted to our hospital because of stable angina pectoris. Coronary angiography revealed $75 \%$ in-stent restenosis at the proximal LAD, showing a fractional flow reserve of 0.74 at the distal LAD (Figure 1). This lesion was successfully treated using excimer laser coronary angioplasty (ELCA) and Xience Alpine ${ }^{\circledast}$ (Abbott Vascular, Santa Clara, CA, USA) stent implantation under optical frequency domain imaging (OFDI) guidance (FastView ${ }^{\circledast}$, Terumo, Tokyo, Japan) which demonstrated a cavity due to plaque rupture with thrombi, and fibroatheroma (Figure $\mathbf{2 A}$ and $\mathbf{B}$ ). We performed coronary angioscopy (Forwardlooking ${ }^{\circledR}$, OVALIS, Osaka, Japan) pre and post-ELCA for further evaluation with direct vision. Pre-ELCA coronary angioscopy showed a cavity due to plaque rupture with thrombi, indicating the progression of in-stent neoatherosclerosis (Figure 2C). Incomplete stent coverage was confirmed at the stent's proximal segment (Figure 2D). We performed ELCA 6 times using a 1.4-mm concentric laser catheter $\left(\mathrm{CVX} 300^{\circledR}\right.$, Spectranetics, Colorado Springs, CO, USA) at a pulse rate $25 \mathrm{~Hz}$, and energy output $45 \mathrm{~mL} / \mathrm{mm}^{2}$. OFDI detected the ablation of in-stent surficial fibrous plaque after ELCA (Figure 2E and F). Coronary angioscopy revealed neointimal minor bleeding, and stent strut with neointima peeled off due to ELCA (Figure $\mathbf{2 G}$ and $\mathbf{H}$ ). Final coronary angiography showed optimal

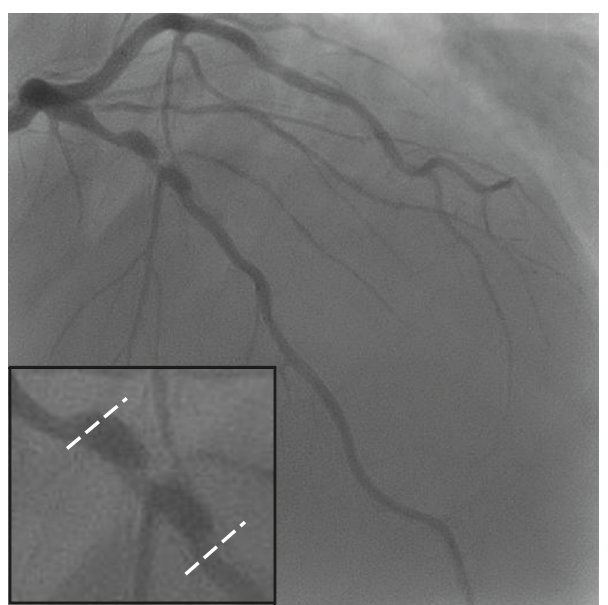

Figure 1. Initial coronary angiography shows $75 \%$ in-stent restenosis at the proximal left anterior descending artery. White dotted lines indicate the stent segment. 

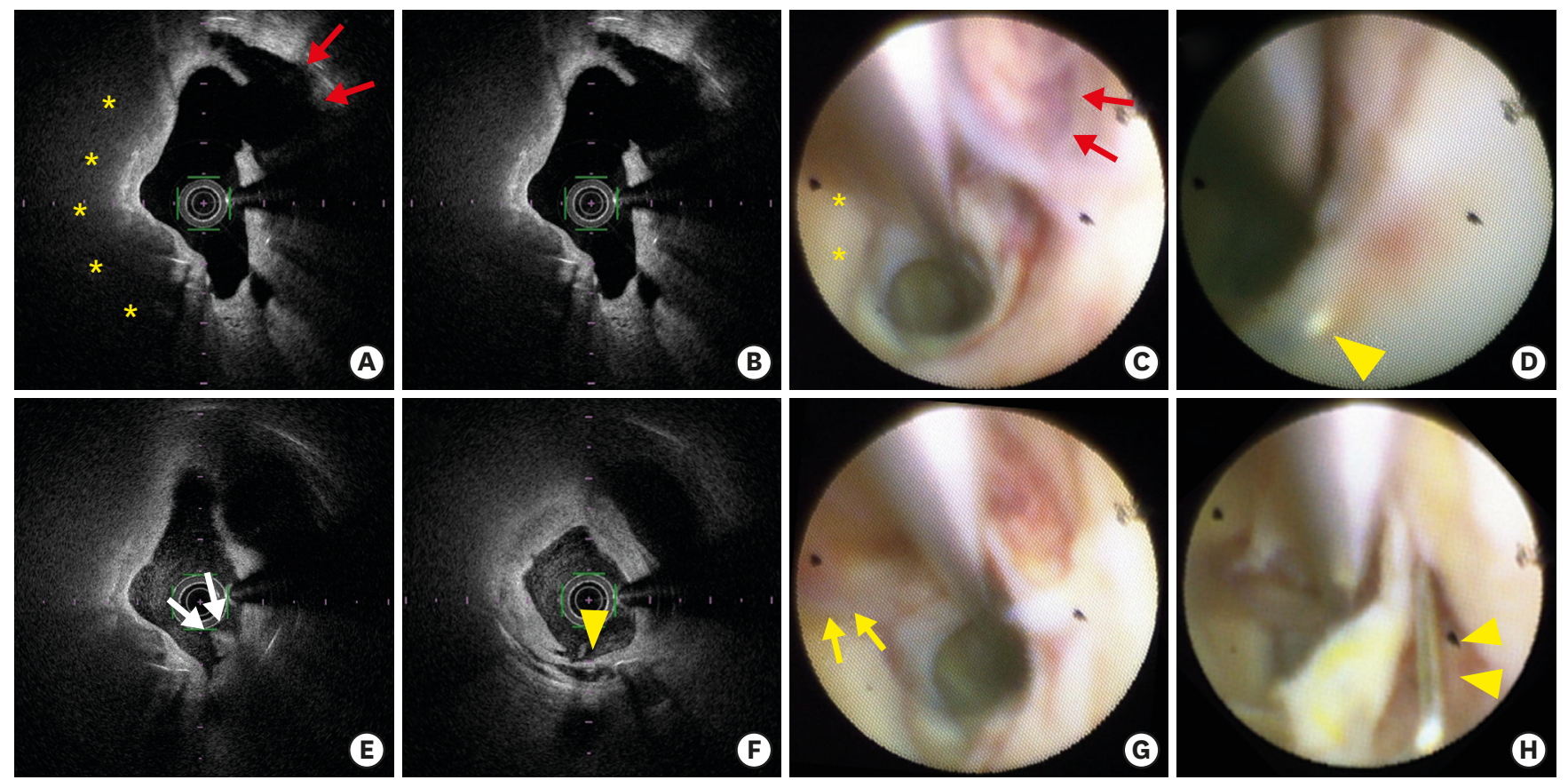

Figure 2. Optical frequency domain imaging and coronary angioscopy findings before excimer laser coronary angioplasty (A-D), and after excimer laser coronary angioplasty (E-H). (A) A cavity (red arrows) and fibroatheroma (yellow asterisks). (B) Circumferential fibrous plaque with a minimum lumen area of $2.8 \mathrm{~mm}^{2}$. (C) On angioscopy, a cavity due to plaque rupture is confirmed at the 1-2 o'clock (red arrows) position, and yellow plaque is confirmed at the 9 o'clock position (yellow asterisks). (D) Yellow arrowhead indicates stent strut. (E) On optical frequency domain imaging, ablation of the surficial plaque is confirmed (white arrows). (F) Ablation of surficial fibrous plaque is confirmed (yellow arrowhead). The minimum lumen area is $2.9 \mathrm{~mm}^{2}$. (G) On angioscopy, surficial minor bleeding is confirmed at the 9 o'clock position (yellow arrows). (H) Yellow arrowheads indicate the stent strut with neointima peeled off.

results (Figure 3). Post-ELCA OFDI demonstrated that ablation of superficial plaque in in-stent area. Following OFDI, coronary angioscopy demonstrated surficial minor bleeding that was unclear on OFDI. Furthermore, coronary angioscopy clearly revealed the exposed strut after ELCA. Clinical studies using ELCA for in-stent restenosis have been reported ${ }^{122)}$; however, coronary angioscopy pre- and post-ELCA is unreported.

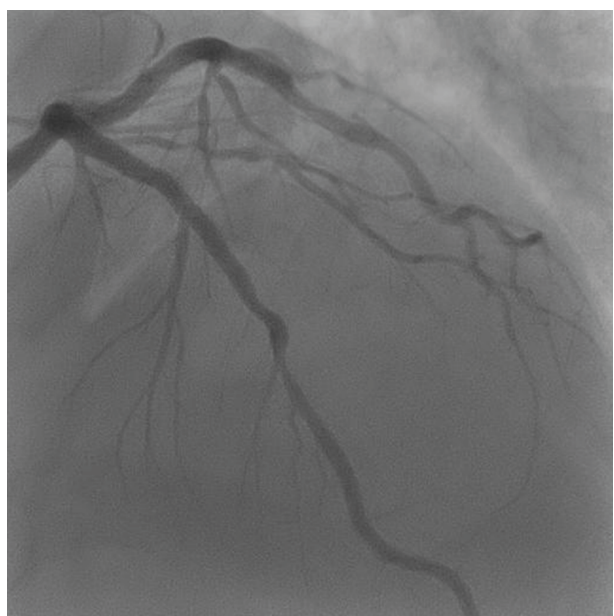

Figure 3. Final coronary angiography shows optimal results with no flow limitation. 


\section{REFERENCES}

1. Ambrosini V, Golino L, Niccoli G, et al. The combined use of drug-eluting balloon and excimer laser for coronary artery restenosis in-stent treatment: the DERIST study. Cardiovasc Revasc Med 2017;18:165-8. PUBMED | CROSSREF

2. Hirose S, Ashikaga T, Hatano Y, et al. Treatment of in-stent restenosis with excimer laser coronary angioplasty: benefits over scoring balloon angioplasty alone. Lasers Med Sci 2016;31:1691-6.

PUBMED | CROSSREF 